\title{
Retroperitoneal approach for robot-assisted partial nephrectomy: a step-by-step description of surgical technique
}

\author{
Alberto Bianchi ${ }^{1}$, Francesco Cianflone ${ }^{1}$, Filippo Migliorini ${ }^{1}$, Maria Angela Cerruto ${ }^{1}$, Alessandro Tafuri ${ }^{1,2}$, \\ Alessandro Antonelli ${ }^{1}$ \\ 'Department of Urology, University of Verona, Azienda Ospedaliera Universitaria Integrata Verona, Verona 37126, Italy. \\ ${ }^{2}$ Department of Neuroscience, Imaging and Clinical Sciences, G. D'Annunzio University, Chieti 66100, Italy. \\ Correspondence to: Prof. Alessandro Antonelli, Department of Urology, University of Verona, Azienda Ospedaliera Universitaria \\ Integrata Verona, Piazzale Aristide Stefani 1, Verona 37126, Italy. E-mail: alessandro.antonelli@aovr.veneto.it; Dr. Alessandro \\ Tafuri, Department of Urology, University of Verona, Azienda Ospedaliera Universitaria Integrata Verona, Piazzale Aristide \\ Stefani 1, Verona 37126, Italy. E-mail: alessandro.tafuri@univr.it
}

How to cite this article: Bianchi A, Cianflone F, Migliorini F, Cerruto MA, Tafuri A, Antonelli A. Retroperitoneal approach for robot-assisted partial nephrectomy: a step-by-step description of surgical technique. Mini-invasive Surg 2021;5:37. https://dx.doi.org/10.20517/2574-1225.2021.64

Received: 10 May 2021 First Decision: 9 Jun 2021 Revised: 16 Jun 2021 Accepted: 23 Jun 2021 First online: 2 Jul 2021

Academic Editors: Michele Marchioni, Luigi Schips Copy Editor: Yue-Yue Zhang Production Editor: Yue-Yue Zhang

\begin{abstract}
In the last decades, minimally invasive partial nephrectomy (PN) has gained traction and, as of today, robotassisted laparoscopic PN (RAPN) is increasingly being performed; this procedure might be performed with a transperitoneal or retroperitoneal (rRAPN) approach. However, rRAPN is less standardized in the literature. Therefore, we describe our rRAPN technique using a da Vinci Xi Surgical System and four robotic arms. First, with the patient placed in full flank position, the camera port is placed at the level of the Petit's triangle apex. Retroperitoneal space is created by turning the index finger in a $180^{\circ}$ movement through this port. After, the two first $8 \mathrm{~mm}$ robotic ports are blindly placed with the surgeon's index finger guide, $8 \mathrm{~cm}$ far from the first port, respectively along the anterior and posterior axillary line; $3-5 \mathrm{~cm}$ caudally to the last one, a $12 \mathrm{~mm}$ AirSeal ${ }^{\circledR}$ assistant port is placed in the same manner. To create space for the last $8 \mathrm{~mm}$ robotic port, the peritoneum is reflected medially and downward off of the transversus abdominis muscle laparoscopically. Only then, the last port is placed under direct vision $8 \mathrm{~cm}$ ventral and about $2 \mathrm{~cm}$ cephalad from the port on the anterior axillary line. The robotic ports placement will result in a caudally convex arc. This technique, due to the extensive use of the surgeon index, implies fast access to the retroperitoneum, protects the underlying anatomical structures from damage, and, due to the trocar positioning along an arc, lowers the arm conflict risk.
\end{abstract}


Keywords: Renal cancer, robotic partial nephrectomy, nephron sparing surgery, retroperitoneal access

\section{INTRODUCTION}

Open partial nephrectomy $(\mathrm{PN})$ has long been the gold standard for treatment of renal masses amenable to nephron-sparing surgery. However, in the last decades, minimally invasive approaches have gained traction in this field, due to improved postoperative recovery without compromised functional, perioperative, and oncological outcomes ${ }^{[1]}$.

Minimally invasive PN may be performed either with laparoscopy (LPN) or with robot-assisted laparoscopy (RAPN); due to the highly advanced laparoscopic skills needed for LPN, RAPN is increasingly being performed, with reports in the literature of shorter warm ischemia time, length of stay, blood loss, and superior functional and oncological outcomes with the latter ${ }^{[2,3]}$.

As with standard laparoscopic techniques, RAPN might be performed with either transperitoneal $(\text { tRAPN })^{[4]}$ or retroperitoneal (rRAPN) approach ${ }^{[5,6]}$.

No specific indication of in which candidates tRAPN or rRAPN should be used can be found in current guidelines, and in the literature the two approaches have been shown to offer equivalent perioperative morbidity, functional and pathological outcomes regardless of tumor location ${ }^{[7,8]}$. However, the choice of surgical approach is influenced by tumor location: tRAPN for medial and anterior masses and rRAPN for posterior ones.

The three- and four-arm RAPN techniques are well described in the literature ${ }^{[9-12]}$. However, a retroperitoneal robotic access technique is less standardised. Therefore, we describe our rRAPN access technique step-by-step, showing all relevant details in the available video [Supplementary Video 1], focusing on patient positioning, port placement, generating retroperitoneal space, and robot docking.

\section{METHODS}

\section{Patient preparation}

For retroperitoneal approaches, bowel preparation is not administered and fasting is indicated from midnight. A type and screen is sent, and two packs of red blood cells are available in the operating room, as for all renal surgeries performed in our department.

\section{Patient positioning}

After general anesthesia is established, the patient is positioned in a full flank position with the ipsilateral side up relative to the renal tumor and the arms extended on supports to facilitate retroperitoneal access. The bed is bent to widen maximally the distance between the iliac crest and the ribs and, eventually, flipped to the anti-Trendelemburg position, in the case of a particularly prominent iliac crest (typically in women) [Figure 1].

Next, after disinfection, surgical drapes are positioned along the paravertebral line laterally and the parasternal line medially, just under the basisternal line cranially and the bisiliac line caudally, in order to provide full access to the retroperitoneal space, as well as exposure of the whole abdomen in case of need to convert to a transperitoneal approach. 


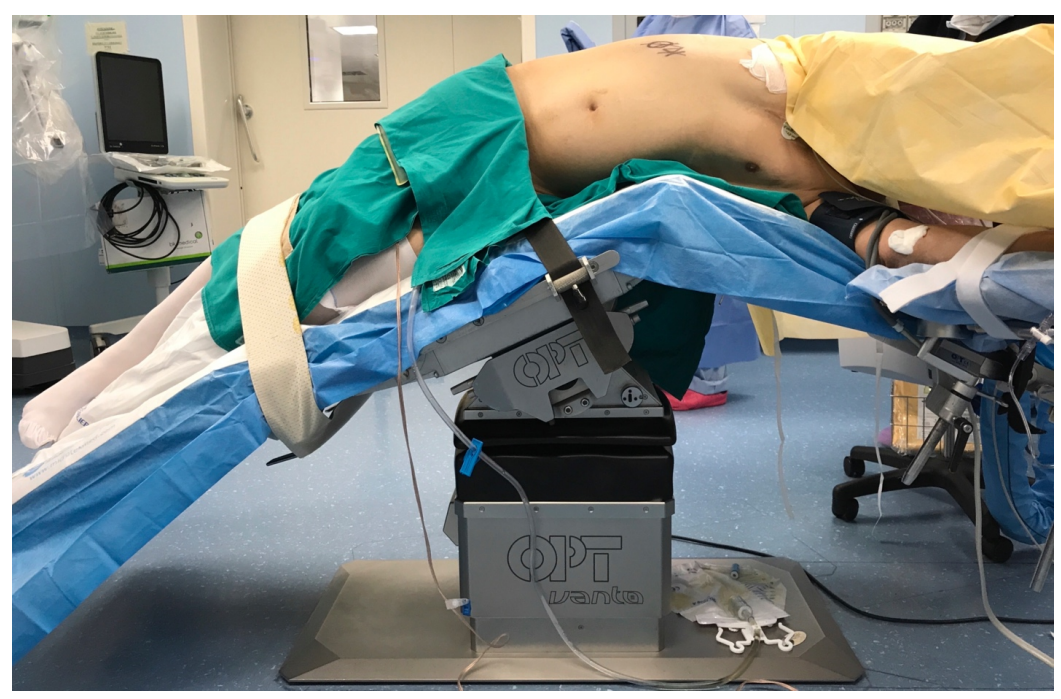

Figure 1. Full flank position and bent bed to increase the distance between the iliac crest and the ribs.

\section{Access}

To identify placement of the camera port and creation of the retroperitoneal space, the iliac crest, 12th rib, and the inferior lumbar (Petit's) triangle are important landmarks and can be marked out [Figure 2A]. A 1.5 $\mathrm{cm}$ vertical incision is made at the level of the apex of the Petit's triangle. Subcutaneous tissue is divided with cautery until the internal oblique muscle is reached. Thereafter, the muscle fibers of the internal oblique muscle are bluntly finger-separated, and then Metzenbaum scissors are used to penetrate the thoracolumbar and trasversalis fasciae and enter the retroperitoneal space [Figure 2B].

\section{Retroperitoneal space creation and port placement}

After inserting the index finger into the previous incision, the retroperitoneal space between the posterior layer of renal fascia and the transversalis fascia is created by turning the index finger in a $180^{\circ}$ movement, running it as close as possible to the abdominal wall, separating the pararenal fat and peritoneum from the transversalis fascia [Figure 3]. During this maneuver, the sensation of the finger running on a smooth surface (the transversalis fascia) and the palpation of the internal surface of the 12th rib and the body of the psoas muscle are crucial to ensure that the surgeon is developing the right space. In case these internal and haptic landmarks are not perceived, the finger could be in the wrong place, such as in between the muscles or inside the peritoneal cavity.

Then, the first two $8 \mathrm{~mm}$ robotic ports are placed at a distance of $8 \mathrm{~cm}$ from the first access port - it generally corresponds to one-finger length - one along the anterior axillary line, the other along the posterior axillary line, $1-2 \mathrm{~cm}$ cranially to the level of the camera port. A $12 \mathrm{~mm}$ AirSeal $^{\circ}$ assistant port is placed on the posterior axillary line, $3-5 \mathrm{~cm}$ caudally to the $8 \mathrm{~mm}$ robotic port [Figure $2 \mathrm{C}-\mathrm{E}$ ]. These first three trocars are bluntly positioned in a "blind fashion", keeping the index finger through the first access port inside the retroperitoneal space, pushing on the abdominal wall at the site of trocar insertions. In this way, the positioning is both fast and safe, although blind, because the internal finger guarantees that nothing else than the abdominal wall is along the route of the trocar. After the insertion of the two first $8 \mathrm{~mm}$ robotic trocars and the AirSeal ${ }^{\circ}$ trocar, the $8 \mathrm{~mm}$ robotic camera port, through a Hasson cone, is placed in the first incision. Then, pneumoretroperitoneum is created at $12 \mathrm{mmHg}$ of carbon dioxide and the $0^{\circ}$ robotic camera can be inserted in the retroperitoneal cavity. 

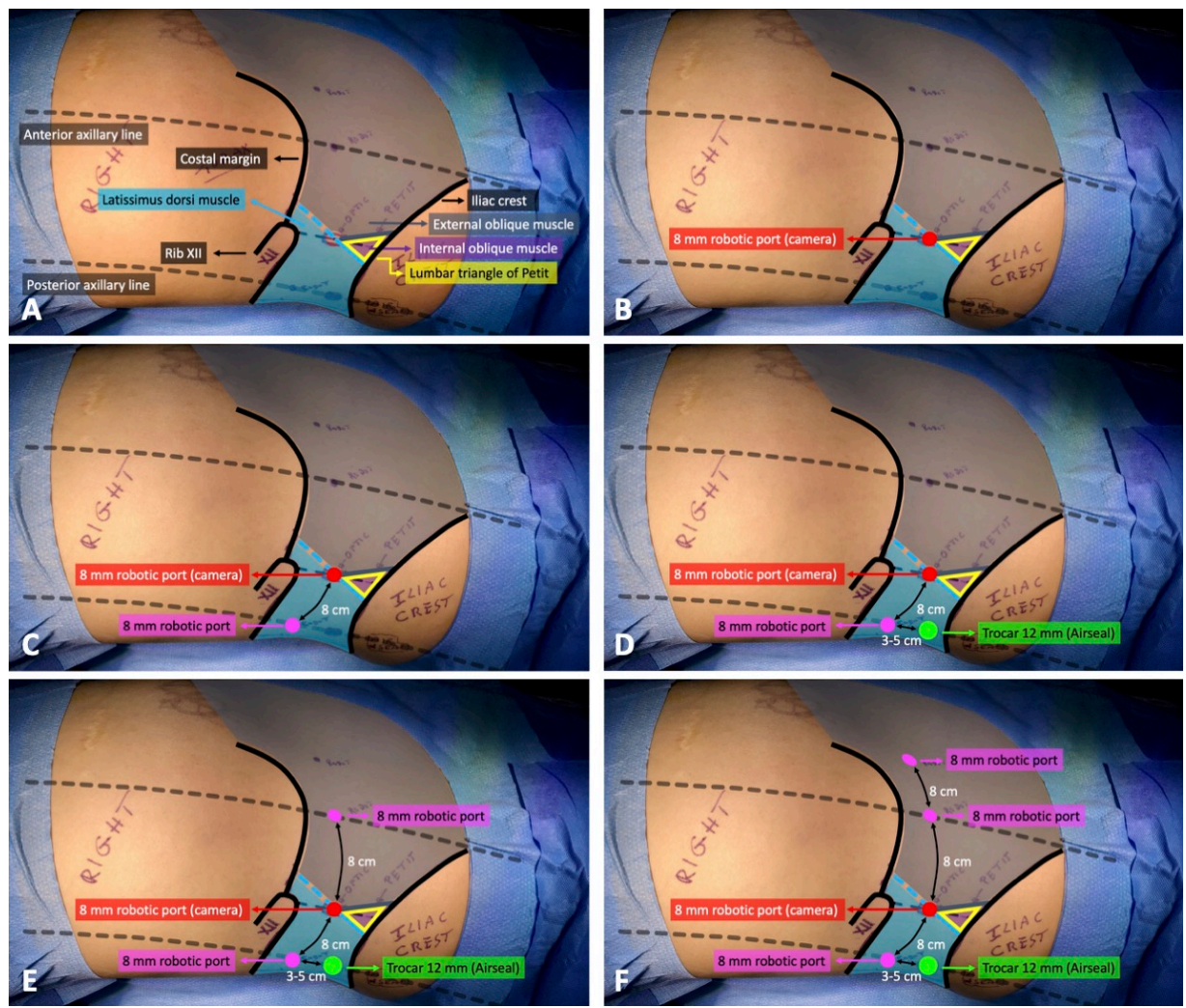

Figure 2. Port placement sequence. (A) The iliac crest, 12th rib, and inferior lumbar (Petit's) triangle are marked. (B) A $1.5 \mathrm{~cm}$ vertical incision is made at the level of the apex of the Petit's triangle for the $8 \mathrm{~mm}$ robotic camera port. (C) Finger-guided $8 \mathrm{~mm}$ robotic port placement along the posterior axillary line. (D) Finger-guided $12 \mathrm{~mm}$ AirSeal assistant port placement along the posterior axillary line. (E) Finger-guided $8 \mathrm{~mm}$ robotic port placement along the anterior axillary line. (F) Under vision, $8 \mathrm{~mm}$ robotic port placement $8 \mathrm{~cm}$ ventrally to the anterior axillary line.

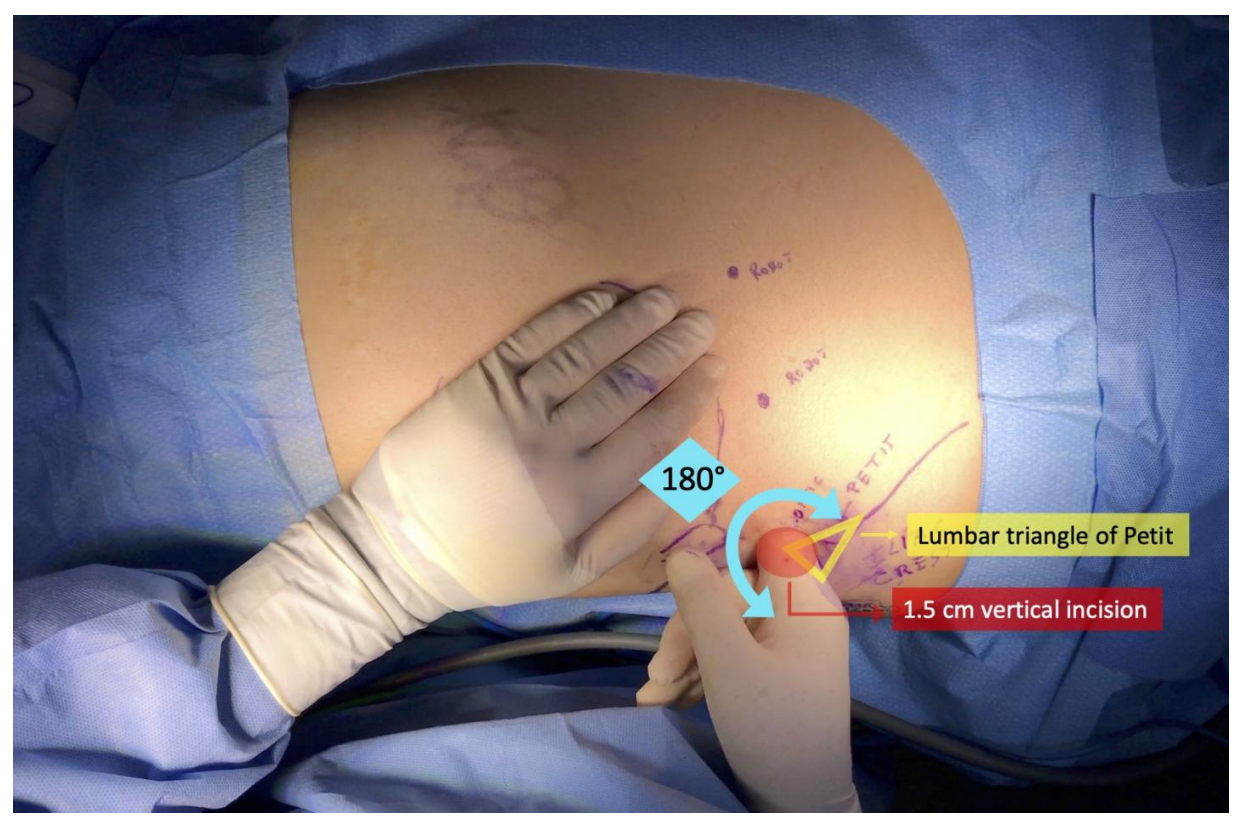

Figure 3. Retroperitoneal space creation: $180^{\circ}$ index finger movement, inserted into the incision at the level of the apex of the Petit's triangle, to separate the perirenal fat and peritoneum from the trasversalis fascia. 
Thereafter, blunt laparoscopic instruments, placed into the AirSeal ${ }^{\circ}$ assistant port and the $8 \mathrm{~mm}$ robotic port in the posterior axillary line, are used to gently reflect the peritoneum medially and downward from the transversus abdominis muscle and allow the creation of space anteriorly for the fourth $8 \mathrm{~mm}$ robotic port [Figure 4].

This is placed under direct vision along the other robotic ports arc, $8 \mathrm{~cm}$ ventral and about $2 \mathrm{~cm}$ cephalad from the one placed on the anterior axillary line [Figures $2 \mathrm{~F}$ ]. A needle can be inserted to confirm the site before the port is inserted under vision.

Particular care should be taken during this critical step to avoid breaching the peritoneum. If this does happen, surgery may still proceed aided by using a th robotic arm by retracting anteriorly the kidney and plugging the opening; alternatively, the breaching can be widely opened and surgery converted to a posteriorly-approached transperitoneal procedure.

The final aspect of the robotic ports placement will result in a caudally convex arc, which creates generous movement space for the four robotic arms (the camera port and the three robotic arms), as well as for the bedside assistant surgeon [Figures 5-7].

\section{Robot docking}

Using the da Vinci Xi Surgical System with a rotating boom, the robot can be brought in at several different locations and the tower rotated to align with the trocars. In our practice, the patient cart is brought in from the patient ventral side, the boom is extended, the camera is docked, the target surgical site is confirmed, the Xi system automatically calibrates the arms, and then we dock them. Once docked, the robotic instruments are inserted under direct vision starting from the fourth arm to facilitate the vision: Maryland bipolar forceps in the left hand, monopolar curved scissors in the right hand, and large needle driver in the fourth $\mathrm{arm}$. The assistant access to the kidney is through the $12 \mathrm{~mm}$ Airseal ${ }^{\circ}$ assistant port [Figure 8].

\section{Surgical technique}

The first step consists in dissecting the pararenal fat to expose the psoas muscle and the posterior layer of renal fascia [Figure 9A]. The next step is to make an incision in the posterior layer of renal fascia just above and parallel to the psoas muscle, exposing the perirenal fat [Figure $9 \mathrm{~B}$ and $\mathrm{C}$ ]. At this point, perirenal fat is dissected from the kidney following the plane along the psoas muscle, which is exposed by retracting anteriorly the kidney using the 4 th arm [Figure $9 \mathrm{D}$ ]. When a perirenal fat pulsation is found, the renal vascular hilum can be easily identified [Figure $9 \mathrm{E}$ and $\mathrm{F}$ ], thus the rest of the operation follows the standard tRAPN steps. At the end, the specimen is retrieved through the camera port.

\section{DISCUSSION}

Since the first laparoscopic nephrectomy by Clayman et al. ${ }^{[13]}$ in 1991 and a few years after the retroperitoneal approach by Gaur et al. ${ }^{[14]}$ were described, a debate about the different minimally invasive $\mathrm{PN}$ approaches was started, and it is still going on as of today.

Selection of the optimal approach plays a critical role in renal surgery. This must be guided by several aspects including the surgeon's experience, the characteristic and location of the kidney mass, and the patient's characteristics and clinical history. Due to larger intra-abdominal space and familiar anatomical landmarks, the transperitoneal access may especially be attractive at the start of the surgeon's experience in this field. On the other hand, a smaller working space and the absence of anatomic landmarks, which limit retroperitoneal approaches, may disorient the beginner surgeon ${ }^{[15]}$. In addition, limited working space 


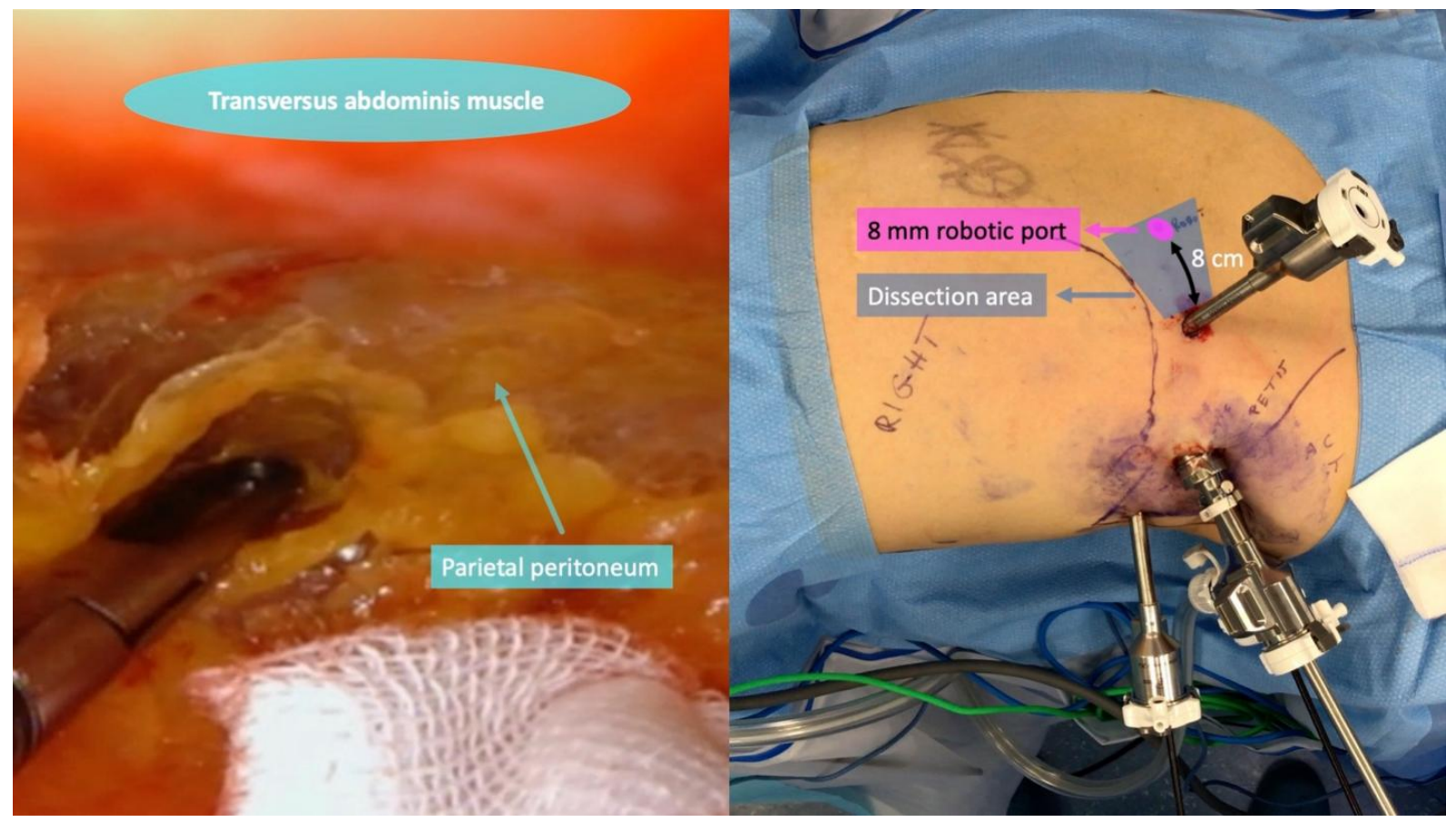

Figure 4. Ventral laparoscopic blunt dissection of the perirenal fat and peritoneum to create space for the fourth port.

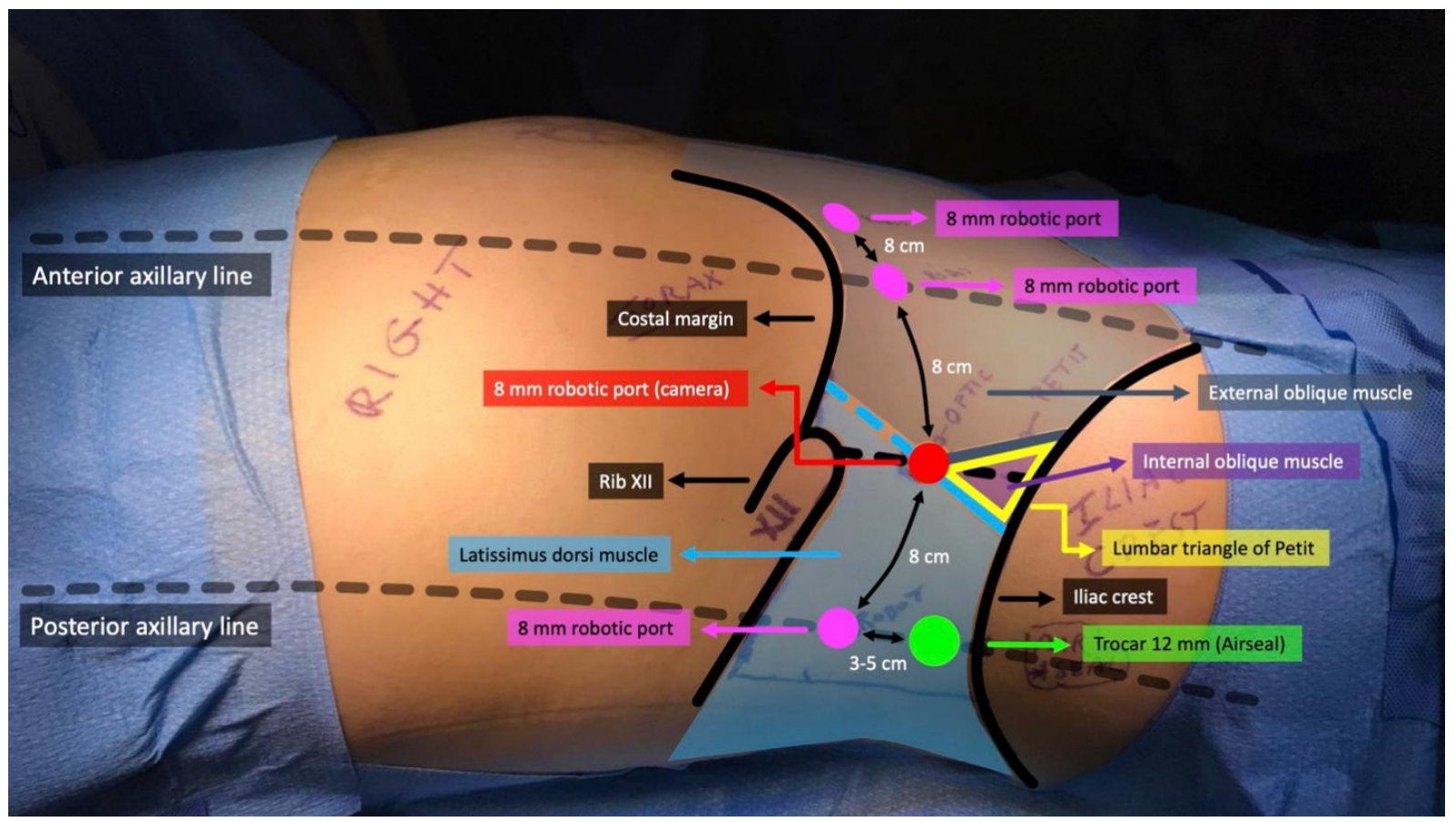

Figure 5. Port configuration for retroperitoneal robot-assisted partial nephrectomy (full lateral view).

reduces triangulation and freedom of movement and increases instrument clashing, making it difficult to use a 4 th robotic $\operatorname{arm}^{[16]}$. 


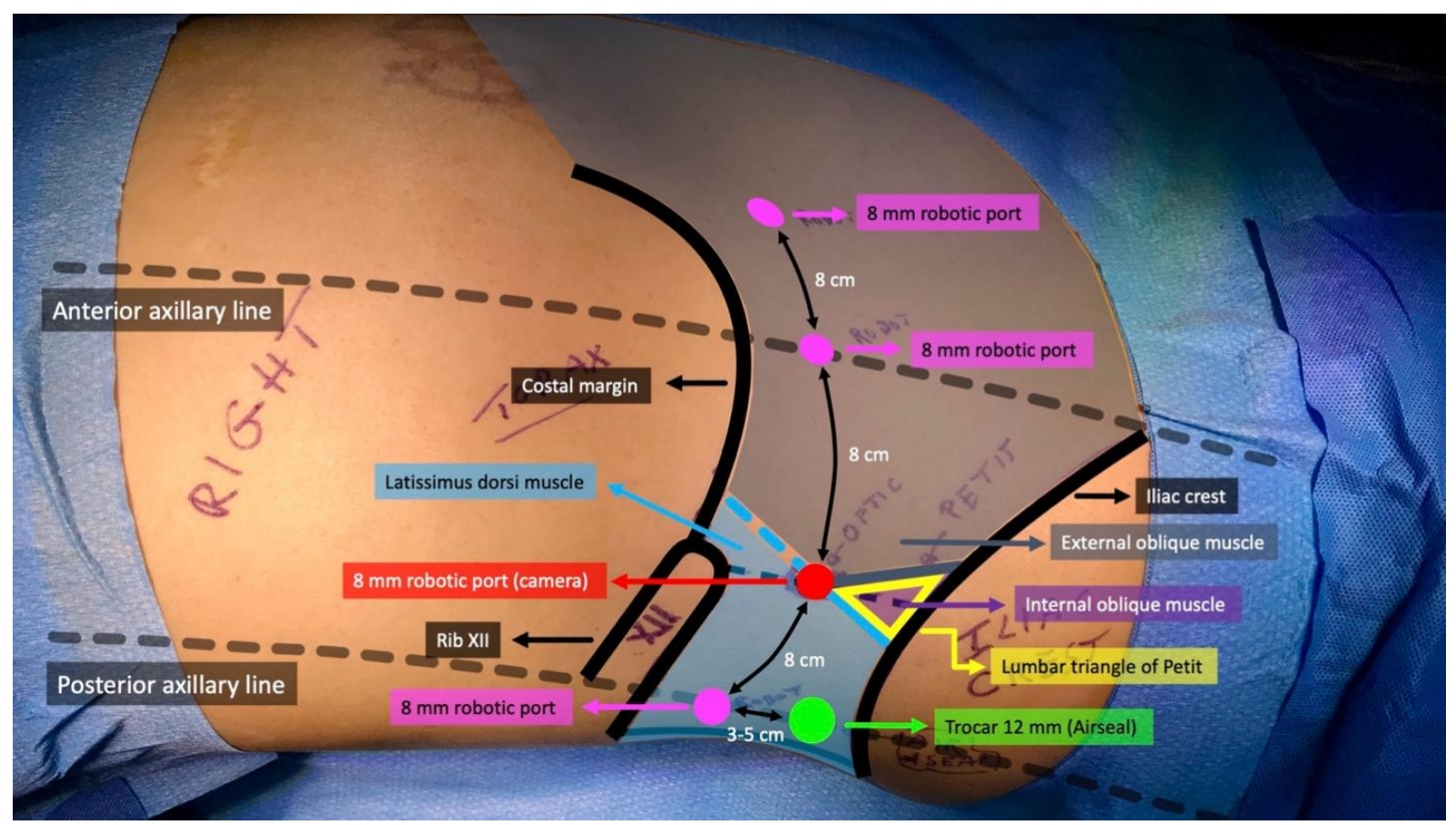

Figure 6. Port configuration for retroperitoneal robot-assisted partial nephrectomy (antero-lateral view).

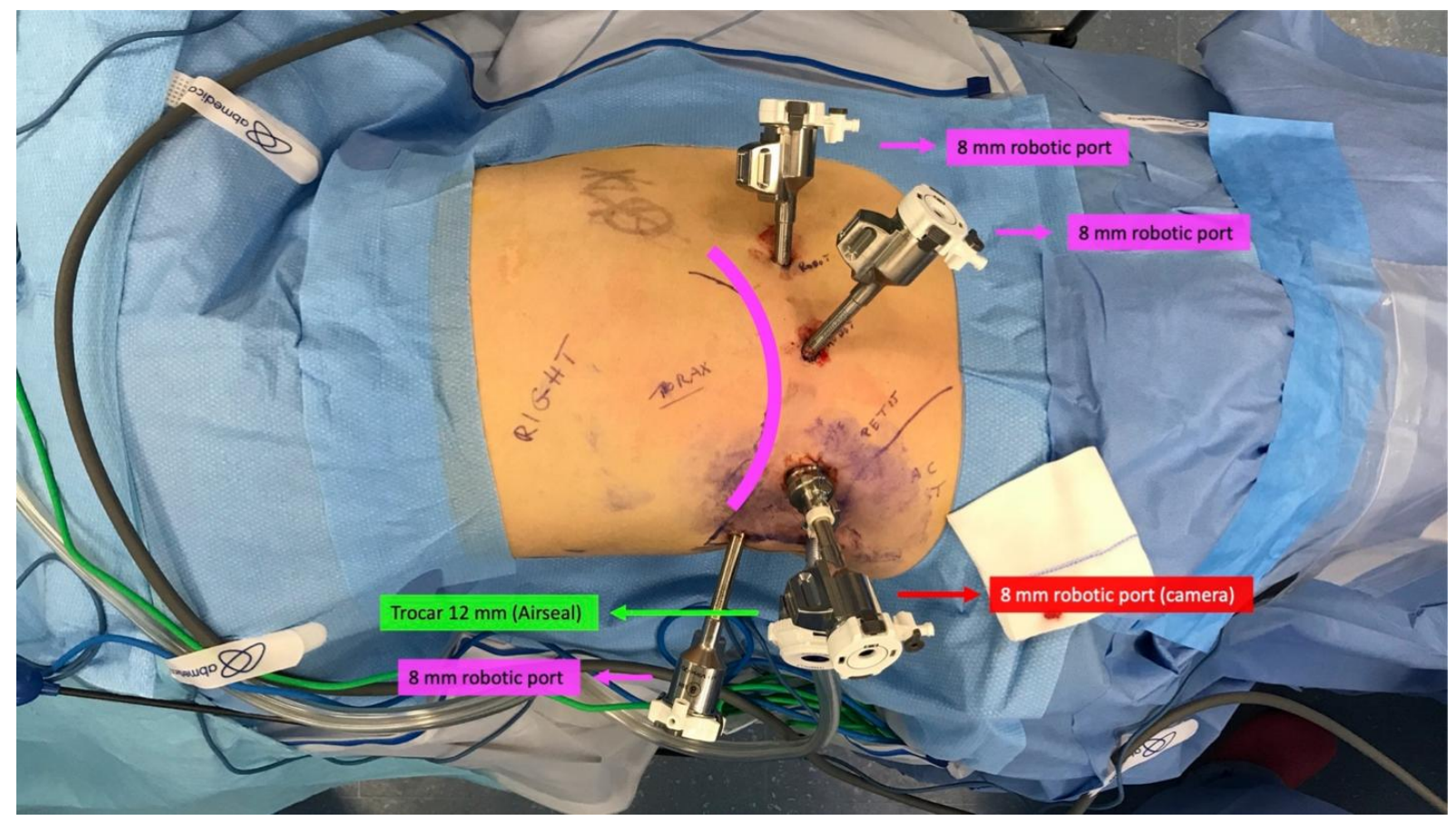

Figure 7. Final port placement delineates a caudally convex arc.

Since it was first described by Patel et al. ${ }^{[5]}$ in 2009, rRAPN has provided a quick and direct access to the renal artery, encountered before the renal vein, without the need for colon mobilization, thus reducing the risk of ileus and with the advantage of faster recovery of postoperative bowel function ${ }^{[16-19]}$. A further potential benefit is that the retroperitoneal space may tamponade hemorrhage and prevent peritonitis 


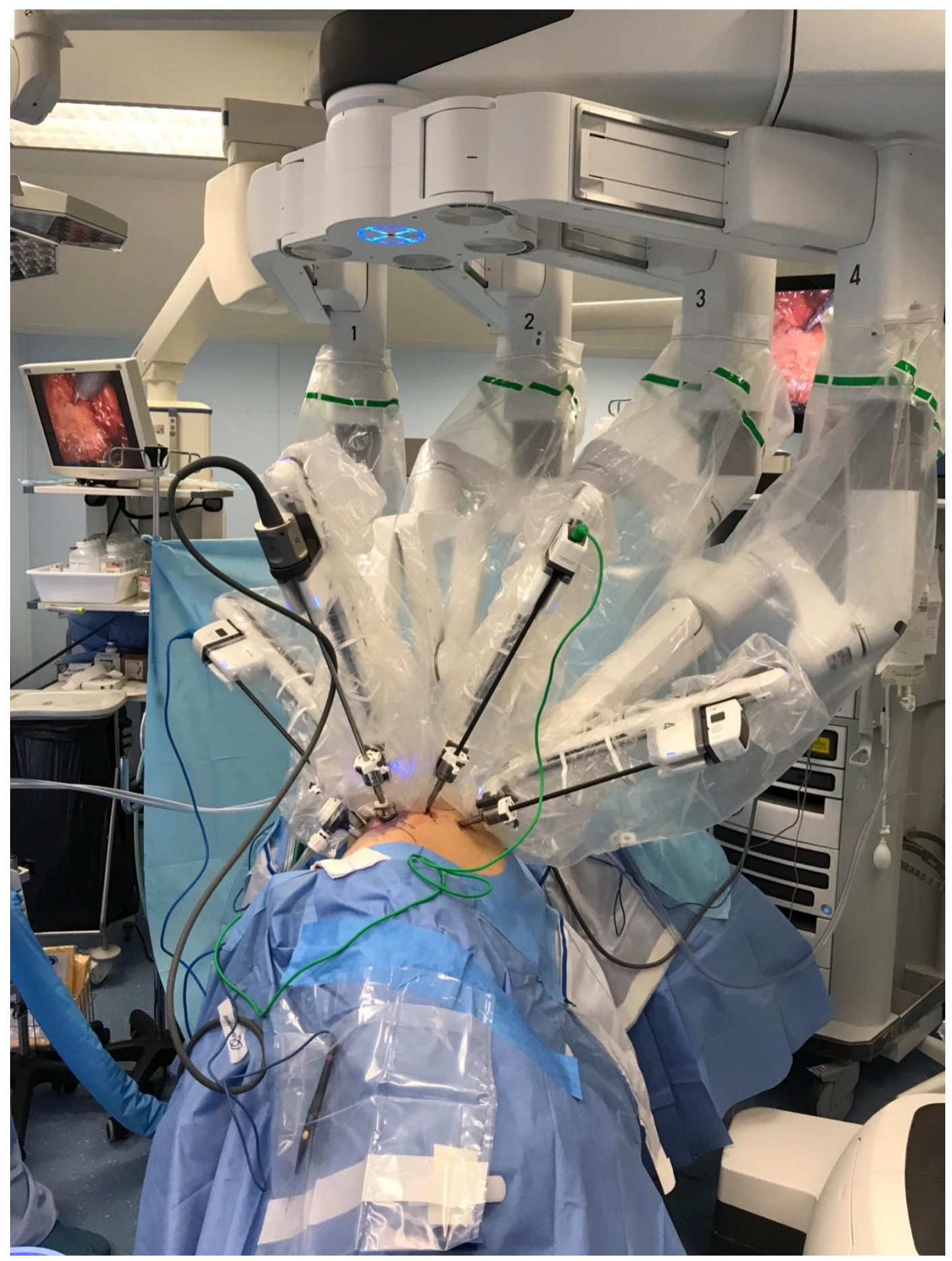

Figure 8. Docked da Vinci Xi Surgical System patient cart, previously positioned ventral to the patient. 

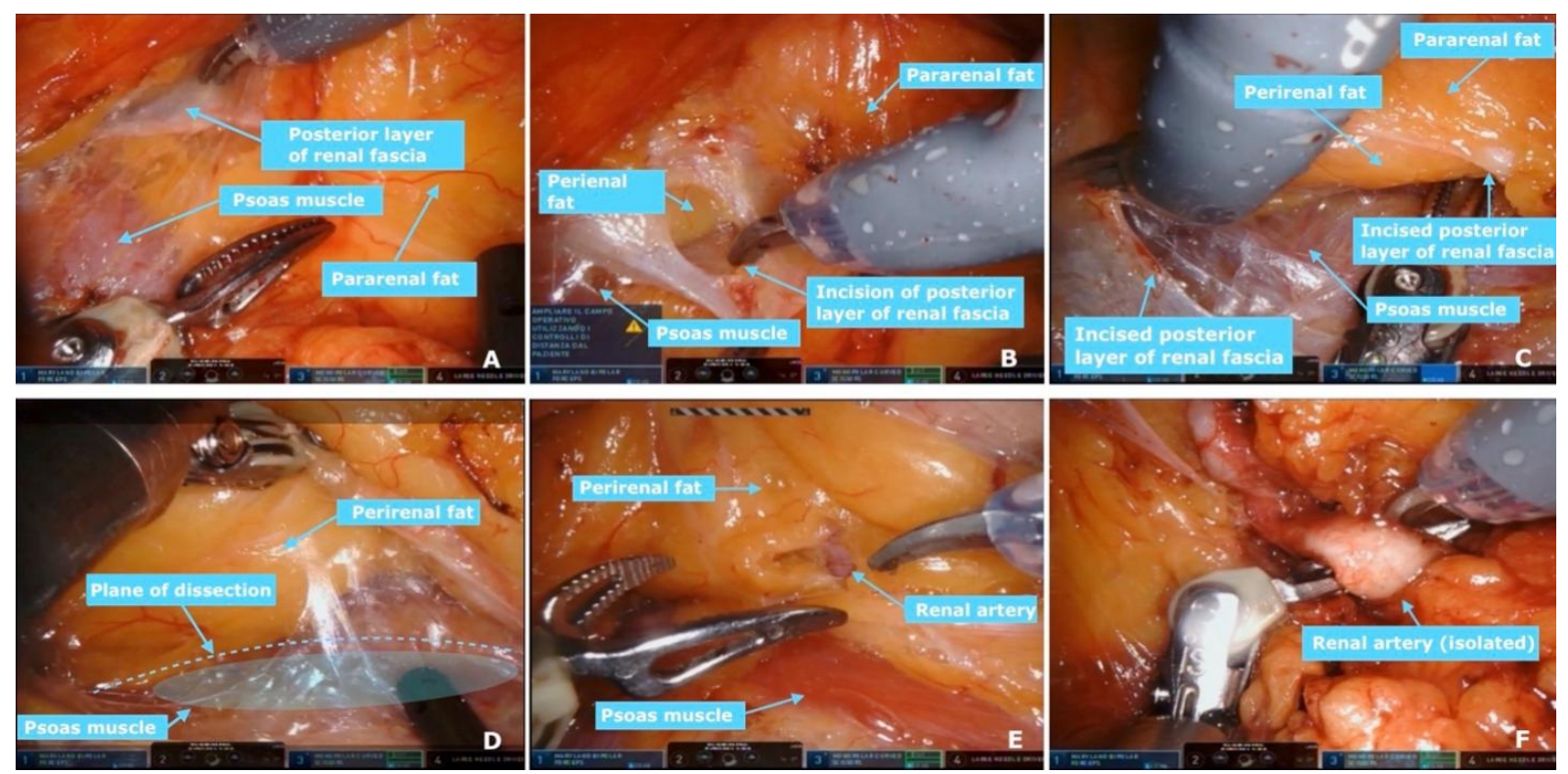

Figure 9. Surgical technique for retroperitoneal robot-assisted partial nephrectomy: (A) dissection of the pararenal fat to expose the psoas muscle and the posterior layer of renal fascia; $(B, C)$ incision in the posterior layer of renal fascia just above and parallel to the psoas muscle, exposing the perirenal fat; (D) dissection of the perirenal fat from the kidney following the plane along the psoas muscle; and $(E, F)$ isolation of the renal artery from the perirenal fat.

caused by urinary fistulas ${ }^{[16]}$.

The significance of tumor location on treatment choice is supported by the categorization of anterior and posterior location by both the RENAL and PADUA scores ${ }^{[20,21]}$. rRAPN is ideally suited for posterior or lateral renal masses, especially in the middle and upper pole of the kidney, because it allows direct access to the posterior and lateral surface of the kidney and minimizes the extent of dissection inside renal fascia, but it can be applied to anterior and medial masses in patients with a history of extensive previous abdominal surgery and/or any pathological condition that may increase the risk of intra-abdominal scarring and adhesions (e.g., previous peritoneal pathology or peritonitis and peritoneal dialysis) ${ }^{[16]}$ bearing in mind that, as stated above, rRAPN represents only an alternative approach for posterior renal masses. Thus, the surgeon's experience and not the tumor location cover a primary role during selection of the optimal surgical approach for posterior masses ${ }^{[7,8]}$.

Previous history of retroperitoneal surgery or percutaneous procedures represents a relative contraindication to rRAPN, as well as highly complex tumors and anatomical variations (e.g., horseshoe kidney and pelvic kidney). Extremely obese patients are more difficult to treat retroperitoneally due to the high volume of adherent perirenal fat and a transperitoneal approach should be preferred ${ }^{[15,16]}$.

Our retroperitoneal space creation technique, due to the extensive use of the surgeon index to guide the procedure, implies fast access to the retroperitoneum. Moreover, the tactile feedback from the surgeon index provides insight on the tissues' characteristics, rendering the blunt dissection of the pararenal fat from the trasversalis fascia almost atraumatic. The trocars' placement with digital protection and feedback is safe and protects the underlying anatomical structures from the trocars' damage, while enabling fast placement. In the literature, retroperitoneal space creation is almost always described using a balloon dissector. The absence of a trocar balloon dilator further reduces the operative time and cost. Meanwhile, placing the 
robotic ports in a caudally convex arc, $8 \mathrm{~cm}$ apart, lowers the risk of arm conflict. This robotic port configuration was already described by Mittakanti et al. ${ }^{[22]}$ reporting the rRAPN technique using the da Vinci Xi Surgical System. Conversely, placing the assistant port dorsally and not ventrally enables always having two instruments ventral (the two $8 \mathrm{~mm}$ robotic ports) and two instruments dorsal (the other $8 \mathrm{~mm}$ robotic port and the $12 \mathrm{~mm}$ AirSeal ${ }^{\circ}$ assistant port) to the camera. This balances retroperitoneal space management and provides, without triangulation, improved freedom of movement for the robotic arms, with two instruments coming from each side, as opposed to other described techniques, where three instruments are brought in anteriorly (two $8 \mathrm{~mm}$ robotic ports and the assistant port), reducing potential clashing of the robotic arms. This is further aided by the da Vinci Xi laser targeting system and automatic arm calibration. In addition, this port configuration gives the assistant more room to work externally and provides an improved angle at which to provide assistance.

Furthermore, the advent of the most recent surgical robot, the single-port da Vinci SP, may facilitate an even more flexible access, even in the field of retroperitoneal renal surgery ${ }^{[23]}$.

\section{Conclusions}

We present in this manuscript a new retroperitoneal access technique for RAPN, developed to facilitate the robotic approach for posterior masses, without any need of a balloon dissector and with better retroperitoneal space management. However, data regarding this technique are still maturing, therefore further studies will be presented in time.

\section{DECLARATIONS}

\section{Authors' contribution}

Study concept and design: Antonelli A, Tafuri A, Bianchi A

Drafting of the manuscript: Bianchi A, Cianflone F

Critical revision of the manuscript for important intellectual content: Antonelli A, Cerruto MA, Migliorini F

Supervision: Antonelli A

\section{Availability of data and materials}

Not applicable.

\section{Financial support and sponsorship}

None.

\section{Conflicts of interest}

All authors declared that there are no conflicts of interest.

\section{Ethical approval and consent to participate}

All images were obtained from the same patient who signed an informed consent.

\section{Consent for publication}

Not applicable.

\section{Copyright}

(c) The Author(s) 2021. 


\section{REFERENCES}

1. Campbell S, Uzzo RG, Allaf ME, et al. Renal mass and localized renal cancer: AUA guideline. J Urol 2017;198:520-9. DOI PubMed

2. Cacciamani GE, Medina LG, Gill T, et al. Impact of surgical factors on robotic partial nephrectomy outcomes: comprehensive systematic review and meta-analysis. J Urol 2018;200:258-74. DOI PubMed

3. Ghani KR, Sukumar S, Sammon JD, Rogers CG, Trinh QD, Menon M. Practice patterns and outcomes of open and minimally invasive partial nephrectomy since the introduction of robotic partial nephrectomy: results from the nationwide inpatient sample. J Urol 2014;191:907-12. DOI PubMed

4. Benway BM, Wang AJ, Cabello JM, Bhayani SB. Robotic partial nephrectomy with sliding-clip renorrhaphy: technique and outcomes. Eur Urol 2009;55:592-9. DOI PubMed

5. Patel MN, Kaul SA, Laungani R, et al. Retroperitoneal robotic renal surgery: technique and early results. J Robot Surg 2009;3:1. DOI PubMed

6. Ghani KR, Porter J, Menon M, Rogers C. Robotic retroperitoneal partial nephrectomy: a step-by-step guide. BJU Int 2014;114:311-3. DOI PubMed

7. Dell'Oglio P, De Naeyer G, Xiangjun L, et al; ERUS Educational Working Group and the YAU working group on robot-assisted surgery. The impact of surgical strategy in robot-assisted partial nephrectomy: is it beneficial to treat anterior tumours with transperitoneal access and posterior tumours with retroperitoneal access? Eur Urol Oncol 2021;4:112-6. DOI PubMed

8. Porpiglia F, Mari A, Amparore D, et al; RECORD 2 Project. Transperitoneal vs retroperitoneal minimally invasive partial nephrectomy: comparison of perioperative outcomes and functional follow-up in a large multi-institutional cohort (The RECORD 2 Project). Surg Endosc 2020. DOI PubMed

9. Feliciano J, Stifelman M. Robotic retroperitoneal partial nephrectomy: a four-arm approach. J Soc Laparoendosc Surg 2012;16:20811. DOI PubMed PMC

10. Hu JC, Treat E, Filson CP, et al. Technique and outcomes of robot-assisted retroperitoneoscopic partial nephrectomy: a multicenter study. Eur Urol 2014;66:542-9. DOI PubMed

11. Gettman M, Blute M, Chow G, Neururer R, Bartsch G, Peschel R. Robotic-assisted laparoscopic partial nephrectomy: technique and initial clinical experience with DaVinci robotic system. Urology 2004;64:914-8. DOI PubMed

12. Singh I. Robot-assisted laparoscopic partial nephrectomy: current review of the technique and literature. J Minim Access Surg 2009;5:87-92. DOI PubMed PMC

13. Clayman RV, Kavoussi LR, Soper NJ, et al. Laparoscopic nephrectomy. N Engl J Med 1991;324:1370-1. DOI PubMed

14. Gaur DD, Agarwal DK, Purohit KC. Retroperitoneal laparoscopic nephrectomy: initial case report. J Urol 1993;149:103-5. DOI PubMed

15. Harke NN, Darr C, Radtke JP, et al. Retroperitoneal versus transperitoneal robotic partial nephrectomy: a multicenter matched-pair analysis. Eur Urol Focus 2020;S2405-4569(20)30254. DOI PubMed

16. Marconi L, Challacombe B. Robotic partial nephrectomy for posterior renal tumours: retro or transperitoneal approach? Eur Urol Focus 2018;4:632-5. DOI PubMed

17. Stroup SP, Hamilton ZA, Marshall MT, et al. Comparison of retroperitoneal and transperitoneal robotic partial nephrectomy for pentafecta perioperative and renal functional outcomes. World J Urol 2017;35:1721-8. DOI PubMed

18. Choo SH, Lee SY, Sung HH, et al. Transperitoneal versus retroperitoneal robotic partial nephrectomy: matched-pair comparisons by nephrometry scores. World J Urol 2014;32:1523-9. DOI PubMed

19. Hu JC, Treat E, Filson CP, et al. Technique and outcomes of robot-assisted retroperitoneoscopic partial nephrectomy: a multicenter study. Eur Urol 2014;66:542-9. DOI PubMed

20. Kutikov A, Uzzo RG. The R.E.N.A.L. nephrometry score: a comprehensive standardized system for quantitating renal tumor size, location and depth. J Urol 2009;182:844-53. DOI PubMed

21. Ficarra V, Novara G, Secco S, et al. Preoperative aspects and dimensions used for an anatomical (PADUA) classification of renal tumours in patients who are candidates for nephron-sparing surgery. Eur Urol 2009;56:786-93. DOI PubMed

22. Mittakanti HR, Heulitt G, Li HF, Porter JR. Transperitoneal vs. retroperitoneal robotic partial nephrectomy: a matched-paired analysis. World J Urol 2020;38:1093-9. DOI PubMed

23. Maurice MJ, Ramirez D, Kaouk JH. Robotic laparoendoscopic single-site retroperitioneal renal surgery: initial investigation of a purpose-built single-port surgical system. Eur Urol 2017;71:643-7. DOI PubMed 